\title{
TWO DISCRETE INEQUALITIES OF GRÜSS TYPE VIA PÓLYA-SZEGÖ AND SHISHA-MOND RESULTS FOR REAL NUMBERS
}

\author{
S. S. DRAGOMIR AND L. KHAN
}

\begin{abstract}
Some new Grüss type discrete inequalities for nonnegative real numbers and applications for the moments of guessing mappings are given.
\end{abstract}

\section{Introduction}

In 1950, Biernacki, Pidek and Ryll-Nardzewski [1] proved the following Grüss type discrete inequality.

If $\overline{\boldsymbol{a}}=\left(a_{1}, \ldots, a_{n}\right)$ and $\overline{\boldsymbol{b}}=\left(b_{1}, \ldots, b_{n}\right)$ are such that there exists the real numbers $a, A, b, B$ with

$$
a \leq a_{i} \leq A, \quad b \leq b_{i} \leq B, \quad i \in\{1, \ldots, n\}
$$

then

$$
\begin{aligned}
\left|C_{n}(\overline{\boldsymbol{a}}, \overline{\boldsymbol{b}})\right| & \leq \frac{1}{n}\left[\frac{n}{2}\right]\left(1-\frac{1}{n}\left[\frac{n}{2}\right]\right)(A-a)(B-b) \\
& =\frac{1}{n^{2}}\left[\frac{n^{2}}{4}\right](A-a)(B-b) \\
& \leq \frac{1}{4}(A-a)(B-b)
\end{aligned}
$$

where

$$
C_{n}(\overline{\boldsymbol{a}}, \overline{\boldsymbol{b}}):=\frac{1}{n} \sum_{i=1}^{n} a_{i} b_{i}-\frac{1}{n} \sum_{i=1}^{n} a_{i} \cdot \frac{1}{n} \sum_{i=1}^{n} b_{i} .
$$

A weighted version of the above result has been obtained in 1988 by Andrica and Badea [2].

Let $\overline{\boldsymbol{a}}, \overline{\boldsymbol{b}}$ satisfy $(1.1)$ and $\overline{\boldsymbol{p}}=\left(p_{1}, \ldots, p_{n}\right)$ be an $n$-tuple of nonnegative numbers with $P_{n}:=\sum_{i=1}^{n} p_{i}>0$. If $S$ is a subset of $\{1, \ldots, n\}$ that minimises the expression

$$
\left|\sum_{i \in S} p_{i}-\frac{1}{2} P_{n}\right|
$$

Received September 5, 2002; revised November 11, 2003.

2000 Mathematics Subject Classification. Primary 26D15; Secondary 94A05.

Key words and phrases. Integral inequalities, Grüss type discrete inequalities, Pólya-Szegö inequality, Shisha-Mond inequality. 
then

$$
\begin{aligned}
C_{n}(\overline{\boldsymbol{p}}, \overline{\boldsymbol{a}}, \overline{\boldsymbol{b}}) & \leq \frac{P_{S}}{P_{n}}\left(1-\frac{P_{S}}{P_{n}}\right)(A-a)(B-b) \\
& \leq \frac{1}{4}(A-a)(B-b),
\end{aligned}
$$

where $P_{S}:=\sum_{i \in S} p_{i}$ and

$$
C_{n}(\overline{\boldsymbol{p}}, \overline{\boldsymbol{a}}, \overline{\boldsymbol{b}}):=\frac{1}{P_{n}} \sum_{i=1}^{n} p_{i} a_{i} b_{i}-\frac{1}{P_{n}} \sum_{i=1}^{n} p_{i} a_{i} \cdot \frac{1}{P_{n}} \sum_{i=1}^{n} p_{i} b_{i} .
$$

Recently, Dragomir and Booth [3] obtained the following result.

If $\overline{\boldsymbol{a}}, \overline{\boldsymbol{b}}$ are real $n$-tuples and $\overline{\boldsymbol{p}}$ is nonnegative with $P_{n}>0$, then

$$
\left|C_{n}(\overline{\boldsymbol{p}}, \overline{\boldsymbol{a}}, \overline{\boldsymbol{b}})\right| \leq \max _{1 \leq j \leq n-1}\left|\Delta a_{j}\right| \max _{1 \leq j \leq n-1}\left|\Delta b_{j}\right| C_{n}(\overline{\boldsymbol{p}}, \overline{\boldsymbol{e}}, \overline{\boldsymbol{e}})
$$

where $\overline{\boldsymbol{e}}=(1,2, \ldots, n)$ and $\Delta a_{j}:=a_{j+1}-a_{j}$ is the forward difference, and $j=1, \ldots, n-1$. Note that

$$
C_{n}(\overline{\boldsymbol{p}}, \overline{\boldsymbol{e}}, \overline{\boldsymbol{e}})=\frac{1}{P_{n}^{2}} \sum_{i=1}^{n} i^{2} p_{i}-\left(\frac{1}{P_{n}} \sum_{i=1}^{n} i p_{i}\right)^{2} .
$$

In particular, we have

$$
\left|C_{n}(\overline{\boldsymbol{a}}, \overline{\boldsymbol{b}})\right| \leq \frac{1}{12}\left(n^{2}-1\right) \max _{1 \leq j \leq n-1}\left|\Delta a_{j}\right| \max _{1 \leq j \leq n-1}\left|\Delta b_{j}\right|
$$

The constant $\frac{1}{12}$ is best possible.

In 2002, Dragomir [4] extended the above result for the $p$-norm. Namely, he proved that

$$
\left|C_{n}(\overline{\boldsymbol{p}}, \overline{\boldsymbol{a}}, \overline{\boldsymbol{b}})\right| \leq \frac{1}{P_{n}^{2}} \sum_{1 \leq j<i \leq n}(i-j)\left(\sum_{k=1}^{n-1}\left|\Delta a_{k}\right|^{p}\right)^{\frac{1}{p}}\left(\sum_{k=1}^{n-1}\left|\Delta b_{k}\right|^{q}\right)^{\frac{1}{q}}
$$

where $p>1, \frac{1}{p}+\frac{1}{q}=1$.

In particular, we have

$$
\left|C_{n}(\overline{\boldsymbol{a}}, \overline{\boldsymbol{b}})\right| \leq \frac{1}{6} \cdot \frac{n^{2}-1}{n}\left(\sum_{k=1}^{n-1}\left|\Delta a_{k}\right|^{p}\right)^{\frac{1}{p}}\left(\sum_{k=1}^{n-1}\left|\Delta b_{k}\right|^{q}\right)^{\frac{1}{q}}
$$

The constant $\frac{1}{6}$ is best possible.

The case of one-norm [5], can be stated as follows:

$$
\left|C_{n}(\overline{\boldsymbol{p}}, \overline{\boldsymbol{a}}, \overline{\boldsymbol{b}})\right| \leq \frac{1}{2} \cdot \frac{1}{P_{n}^{2}} \sum_{i=1}^{n} p_{i}\left(P_{n}-p_{i}\right) \sum_{k=1}^{n-1}\left|\Delta a_{k}\right| \sum_{k=1}^{n-1}\left|\Delta b_{k}\right| .
$$


In particular, we have

$$
\left|C_{n}(\overline{\boldsymbol{a}}, \overline{\boldsymbol{b}})\right| \leq \frac{1}{2}\left(1-\frac{1}{n}\right) \sum_{k=1}^{n-1}\left|\Delta a_{k}\right| \sum_{k=1}^{n-1}\left|\Delta b_{k}\right| .
$$

The constant $\frac{1}{2}$ is sharp.

Another direction was considered by Cerone and Dragomir in [8].

If $\overline{\boldsymbol{a}}, \overline{\boldsymbol{b}}$ are real $n$-tuples and $\overline{\boldsymbol{p}}$ is a positive $n$-tuple and there exists $m, M \in \mathcal{R}$ such that

$$
m \leq a_{i} \leq M
$$

then one has the inequality

$$
\left|C_{n}(\overline{\boldsymbol{p}}, \overline{\boldsymbol{a}}, \overline{\boldsymbol{b}})\right| \leq \frac{1}{2}(M-m) \frac{1}{P_{n}} \sum_{i=1}^{n} p_{i}\left|b_{i}-\frac{1}{P_{n}} \sum_{j=1}^{n} p_{j} b_{j}\right| .
$$

The constant $\frac{1}{2}$ is best possible. In particular, we have

$$
\left|C_{n}(\overline{\boldsymbol{a}}, \overline{\boldsymbol{b}})\right| \leq \frac{1}{2}(M-m) \cdot \frac{1}{n} \sum_{i=1}^{n}\left|b_{i}-\frac{1}{n} \sum_{j=1}^{n} b_{j}\right| .
$$

The constant $\frac{1}{2}$ is best possible.

In this paper we obtain different Grüss type discrete inequalities for nonnegative real numbers by the use of some counterpart results for the Cauchy-Buniakowsky-Schwarz inequality. Application for the moments of guessing mapping are also given.

\section{Discrete Inequalities}

The following Grüss type inequality holds.

Theorem 1. Let $\overline{\boldsymbol{a}}=\left(a_{1}, \ldots, a_{n}\right)$ and $\overline{\boldsymbol{b}}=\left(b_{1}, \ldots, b_{n}\right)$ be two sequences of positive real numbers with

$$
0<a \leq a_{i} \leq A<\infty \text { and } 0<b \leq b_{i} \leq B<\infty \text { for each } i \in\{1, \ldots, n\} .
$$

Then one has the inequality

$$
\left|C_{n}(\overline{\boldsymbol{a}}, \overline{\boldsymbol{b}})\right| \leq \frac{1}{4} \cdot \frac{(A-a)(B-b)}{\sqrt{a A b B}} \frac{1}{n} \sum_{i=1}^{n} a_{i} \cdot \frac{1}{n} \sum_{i=1}^{n} b_{i} .
$$

The constant $\frac{1}{4}$ is best possible in (2.2) in the sense that it cannot be replaced by a smaller constant. 
Proof. We have, by the Cauchy-Buniakowski-Schwarz inequality for double sums, the inequality

$$
\begin{aligned}
\left|C_{n}(\overline{\boldsymbol{a}}, \overline{\boldsymbol{b}})\right| & =\left|\frac{1}{2 n^{2}} \sum_{i, j=1}^{n}\left(a_{i}-a_{j}\right)\left(b_{i}-b_{j}\right)\right| \\
& \leq \frac{1}{2 n^{2}} \sum_{i, j=1}^{n}\left|\left(a_{i}-a_{j}\right)\left(b_{i}-b_{j}\right)\right| \\
& \leq \frac{1}{2 n^{2}}\left[\sum_{i, j=1}^{n}\left(a_{i}-a_{j}\right)^{2} \sum_{i, j=1}^{n}\left(b_{i}-b_{j}\right)^{2}\right]^{\frac{1}{2}} \\
& =\frac{1}{2 n^{2}}\left[4\left[n \sum_{i=1}^{n} a_{i}^{2}-\left(\sum_{i=1}^{n} a_{i}\right)^{2}\right]\left[n \sum_{i=1}^{n} b_{i}^{2}-\left(\sum_{i=1}^{n} b_{i}\right)^{2}\right]\right]^{\frac{1}{2}} \\
& =\left[\frac{1}{n} \sum_{i=1}^{n} a_{i}^{2}-\left(\frac{1}{n} \sum_{i=1}^{n} a_{i}\right)^{2}\right]^{\frac{1}{2}}\left[\frac{1}{n} \sum_{i=1}^{n} b_{i}^{2}-\left(\frac{1}{n} \sum_{i=1}^{n} b_{i}\right)^{2}\right]^{\frac{1}{2}} .
\end{aligned}
$$

Utilising the Pólya-Szegö inequality [19]

$$
1 \leq \frac{\sum_{i=1}^{n} z_{i}^{2} \sum_{i=1}^{n} u_{i}^{2}}{\left(\sum_{i=1}^{n} z_{i} u_{i}\right)^{2}} \leq \frac{1}{4}\left(\sqrt{\frac{M_{1} M_{2}}{m_{1} m_{2}}}+\sqrt{\frac{m_{1} m_{2}}{M_{1} M_{2}}}\right)^{2},
$$

provided $0<m_{1} \leq z_{i} \leq M_{1}<\infty, 0<m_{2} \leq u_{i} \leq M_{2}<\infty, i \in\{1, \ldots, n\}$, we may state that

$$
\frac{n \sum_{i=1}^{n} a_{i}^{2}}{\left(\sum_{i=1}^{n} a_{i}\right)^{2}} \leq \frac{1}{4}\left(\sqrt{\frac{A}{a}}+\sqrt{\frac{a}{A}}\right)^{2}=\frac{1}{4} \cdot \frac{(A+a)^{2}}{a A}
$$

giving

$$
\frac{n \sum_{i=1}^{n} a_{i}^{2}-\left(\sum_{i=1}^{n} a_{i}\right)^{2}}{\left(\sum_{i=1}^{n} a_{i}\right)^{2}} \leq \frac{1}{4} \cdot \frac{(A+a)^{2}}{a A}-1=\frac{(A-a)^{2}}{4 a A}
$$

that is,

$$
n \sum_{i=1}^{n} a_{i}^{2}-\left(\sum_{i=1}^{n} a_{i}\right)^{2} \leq \frac{(A-a)^{2}}{4 a A}\left(\sum_{i=1}^{n} a_{i}\right)^{2} .
$$


In a similar fashion, we obtain

$$
n \sum_{i=1}^{n} b_{i}^{2}-\left(\sum_{i=1}^{n} b_{i}\right)^{2} \leq \frac{(B-b)^{2}}{4 b B}\left(\sum_{i=1}^{n} b_{i}\right)^{2} .
$$

Using (2.3), (2.5) and (2.6), we deduce the desired inequality (2.2).

Now, assume that the inequality in $(2.2)$ holds with a constant $c>0$, i.e.,

$$
\left|C_{n}(\overline{\boldsymbol{a}}, \overline{\boldsymbol{b}})\right| \leq c \frac{(A-a)(B-b)}{\sqrt{a A b B}} \frac{1}{n} \sum_{i=1}^{n} a_{i} \cdot \frac{1}{n} \sum_{i=1}^{n} b_{i} .
$$

If we choose $n=2, a_{1}=b_{1}, a_{2}=b_{2}, a_{1}=a, a_{2}=A$, then from (2.7) we get

$$
\frac{1}{2}\left(a^{2}+A^{2}\right)-\frac{1}{4}(a+A)^{2} \leq c \frac{(A-a)^{2}}{a A} \cdot \frac{(a+A)^{2}}{4}
$$

giving

from where we get

$$
\frac{1}{4}(A-a)^{2} \leq c \frac{(A-a)^{2}}{a A} \cdot \frac{(a+A)^{2}}{4}
$$

$$
a A \leq c(a+A)^{2} \text { for any } 0<a<A<\infty .
$$

Let $a=1-\varepsilon, A=1+\varepsilon$, with $\varepsilon \in(0,1)$. Then from (2.8) we get $1-\varepsilon^{2} \leq 4 c$ for any $\varepsilon \in(0,1)$, which shows that $c \geq \frac{1}{4}$.

Remark 1. We will now compare the inequality (2.2) with the Grüss inequality

$$
\left|C_{n}(\overline{\boldsymbol{a}}, \overline{\boldsymbol{b}})\right| \leq \frac{1}{4}(A-a)(B-b)
$$

provided $a \leq a_{i} \leq A$ and $b \leq b_{i} \leq B, i \in\{1, \ldots, n\}$.

We consider, for $a, b>0$, the quantity

$$
U:=\frac{1}{\sqrt{a A b B}} \cdot \frac{1}{n} \sum_{i=1}^{n} a_{i} \cdot \frac{1}{n} \sum_{i=1}^{n} b_{i}
$$

and we will assume that $a=b, A=B, a_{i}=b_{i}, i \in\{1, \ldots, n\}$. Thus

$$
U=\frac{\left(\sum_{i=1}^{n} a_{i}\right)^{2}}{n^{2} a A}
$$

Choose $n=3, a_{1}=a_{2}=1, a_{3}=x$. Thus $A a=x$ and we have

$$
U(x)=\frac{(x+2)^{2}}{9 x} .
$$

We observe that

$$
U(x)-1=\frac{x^{2}-5 x+4}{9 x}=\frac{(x-1)(x-4)}{9 x},
$$


showing that if $x \in(0,1] \cup[4, \infty), U(x) \geq 1$ while for $x \in(1,4), U(x)<1$.

In conclusion, the bound provided by (2.2) is sometimes better, and at other times, worse than the bound provided by the Grüss inequality.

The second result of Grüss type is embodied in the following theorem.

Theorem 2. Let $\overline{\boldsymbol{a}}=\left(a_{1}, \ldots, a_{n}\right)$ and $\overline{\boldsymbol{b}}=\left(b_{1}, \ldots, b_{n}\right)$ be two sequences of positive real numbers satisfying (2.2). Then one has the inequality

$$
\left|C_{n}(\overline{\boldsymbol{a}}, \overline{\boldsymbol{b}})\right| \leq(\sqrt{A}-\sqrt{a})(\sqrt{B}-\sqrt{b}) \sqrt{\frac{\sum_{i=1}^{n} a_{i}}{n} \cdot \frac{\sum_{i=1}^{n} b_{i}}{n}} .
$$

The constant $c=1$ is the best possible in the sense that it cannot be replaced by a smaller constant.

Proof. We shall use Shisha-Mond's inequality [20]

$$
\frac{\sum_{i=1}^{n} z_{i}^{2}}{\sum_{i=1}^{n} z_{i} y_{i}}-\frac{\sum_{i=1}^{n} z_{i} y_{i}}{\sum_{i=1}^{n} y_{i}^{2}} \leq\left(\sqrt{\frac{M_{1}}{m_{2}}}-\sqrt{\frac{m_{1}}{M_{2}}}\right)^{2},
$$

provided $0<m_{1} \leq z_{i} \leq M_{1}<\infty$ and $0<m_{2} \leq y_{i} \leq M_{2}<\infty$.

If in (2.11) we choose $z_{i}=a_{i}, y_{i}=1$, then we get

$$
0 \leq \frac{1}{n} \sum_{i=1}^{n} a_{i}^{2}-\left(\frac{1}{n} \sum_{i=1}^{n} a_{i}\right)^{2} \leq \frac{\sum_{i=1}^{n} a_{i}}{n}(\sqrt{A}-\sqrt{a})^{2} .
$$

Similarly

$$
0 \leq \frac{1}{n} \sum_{i=1}^{n} b_{i}^{2}-\left(\frac{1}{n} \sum_{i=1}^{n} b_{i}\right)^{2} \leq \frac{\sum_{i=1}^{n} b_{i}}{n}(\sqrt{B}-\sqrt{b})^{2} .
$$

Now, making use of (2.3), (2.12) and (2.13), we obtain the desired inequality (2.10).

To prove the sharpness of the constant, assume that (2.10) holds with a constant $c>0$, i.e.,

$$
\begin{aligned}
\mid \frac{1}{n} \sum_{i=1}^{n} a_{i} b_{i} & -\frac{1}{n} \sum_{i=1}^{n} a_{i} \cdot \frac{1}{n} \sum_{i=1}^{n} b_{i} \mid \\
\leq & c(\sqrt{A}-\sqrt{a})(\sqrt{B}-\sqrt{b}) \sqrt{\frac{\sum_{i=1}^{n} a_{i}}{n} \cdot \frac{\sum_{i=1}^{n} b_{i}}{n}} .
\end{aligned}
$$

If we choose $n=2, a_{1}=b_{1}, a_{2}=b_{2}, a_{1}=a, a_{2}=A$, then from (2.14) we get

$$
\frac{1}{4}(A-a)^{2} \leq c(\sqrt{A}-\sqrt{a})^{2} \cdot \frac{a+A}{2},
$$


that is,

$$
\frac{1}{4}(\sqrt{A}-\sqrt{a})^{2}(\sqrt{A}+\sqrt{a})^{2} \leq c(\sqrt{A}-\sqrt{a})^{2} \cdot \frac{a+A}{2},
$$

giving for any $0<a<A<\infty$ that

$$
(\sqrt{A}+\sqrt{a})^{2} \leq 2 c(a+A) .
$$

If in (2.15) we choose $a=1-\varepsilon, A=1+\varepsilon, \varepsilon \in(0,1)$, we get $(\sqrt{1-\varepsilon}+\sqrt{1+\varepsilon})^{2} \leq 4 c$. Letting $\varepsilon \rightarrow 0+$, we deduce $c \geq 1$, and the theorem is proved.

Remark 2. We shall show that at some times, the Grüss inequality (2.9) is better, and at other times, the inequality $(2.10)$ is better.

If we choose $a_{i}=b_{i},(i=1, \ldots, n), a=b, A=B$, we have to compare

$$
I_{1}:=\frac{1}{4}(A-a)^{2}
$$

with

$$
I_{2}:=(\sqrt{A}-\sqrt{a})^{2} \frac{\sum_{i=1}^{n} a_{i}}{n} .
$$

If we assume that $a=0, A=1$, then

$$
I_{1}=\frac{1}{4}, \quad I_{2}=\frac{\sum_{i=1}^{n} a_{i}}{n} \quad(i=1, \ldots, n)
$$

showing that for $0 \leq a_{i} \leq 1$ with $\frac{\sum_{i=1}^{n} a_{i}}{n}<\frac{1}{4},(2.10)$ is better than the Grüss inequality while for $\frac{\sum_{i=1}^{n} a_{i}}{n}>\frac{1}{4}$, the Grüss inequality provides a better bound.

Remark 3. We will show now the fact that the bounds provided by (2.2) and (2.10) cannot generally be compared.

Assume that $a_{i}=b_{i},(i=1, \ldots, n), a=b, A=b$ and consider

$$
\begin{aligned}
& J_{1}:=\frac{1}{4} \frac{(A-a)^{2}}{a A}\left(\frac{1}{n} \sum_{i=1}^{n} a_{i}\right)^{2} \\
& J_{2}:=(\sqrt{A}-\sqrt{a})^{2} \frac{\sum_{i=1}^{n} a_{i}}{n} .
\end{aligned}
$$

If we choose $a=1, A=4$, we get

$$
J_{1}=\frac{9}{16} x^{2}, \quad J_{2}=x \text { where } x:=\frac{\sum_{i=1}^{n} a_{i}}{n} \in[1,4] .
$$

We observe that $J_{1}-J_{2}=\frac{x(9 x-16)}{16}$ showing that for $x \in\left[1, \frac{16}{9}\right]$ the bound provided by (2.10) is better than the bound provided by $(2.2)$ while for $x \in\left(\frac{16}{9}, 4\right]$, the conclusion is the other way around. 


\section{Applications for Moments of Guessing Mappings}

In 1994, J. L. Massey [14] considered the problem of guessing the value taken on by a discrete random variable $X$ in one trial of a random experiment by asking questions of the form

$$
\text { "Did } X \text { take on its } i^{\text {th }} \text { possible value?" }
$$

until the answer is

$$
\text { "Yes!". }
$$

This problem arises for instance when a cryptologist must try out possible secret keys one at a time after minimising the possibilities by some cryptoanalysis.

Consider a random variable $X$ with finite range $X=\left\{x_{1}, \ldots, x_{n}\right\}$ and distribution $P_{X}\left(x_{k}\right)=p_{k}$ for $k=1,2, \ldots, n$.

A one-to-one function $G: \chi \rightarrow\{1, \ldots, n\}$ is a guessing function for $X$. Thus

$$
E\left(G^{m}\right):=\sum_{k=1}^{n} k^{m} p_{k}
$$

is the $m^{\text {th }}$ moment of this function, provided we renumber the $x_{i}$ such that $x_{k}$ is always the $k^{\text {th }}$ guess.

In [14], Massey observed that, $E(G)$, the average number of guesses, is minimised by a guessing strategy that guesses the possible values of $X$ in decreasing order of probability.

In the same paper [14], Massey proved that

$$
E(G) \geq \frac{1}{4} 2^{H(X)}+1 \text { provided } H(X) \geq 2 \text { bits }
$$

for an optimal guessing strategy, where $H(X)$ is the Shannon entropy

$$
H(X)=-\sum_{i=1}^{n} p_{i} \log _{2}\left(p_{i}\right) .
$$

He also has shown that $E(G)$ may be arbitrarily large when $H(X)$ is an arbitrarily small positive number such that there is no interesting upper bound on $E(G)$ in terms of $H(X)$.

In 1996, Arikan [15] has proved that any guessing algorithm for $X$ obeys the lower bound

$$
E\left(G^{\rho}\right) \geq \frac{\left[\sum_{k=1}^{n} p_{k}^{\frac{1}{1+\rho}}\right]^{1+\rho}}{[1+\ln n]^{\rho}}, \quad \rho \geq 0
$$

while an optimal guessing algorithm for $X$ satisfies

$$
E\left(G^{\rho}\right) \leq\left[\sum_{k=1}^{n} p_{k}^{\frac{1}{1+\rho}}\right]^{1+\rho}, \quad \rho \geq 0 .
$$


In 1997, Boztaş [16] proved that for $m \geq 1$, integer

$$
\begin{aligned}
E\left(G^{m}\right) \leq & \frac{1}{m+1}\left[\sum_{k=1}^{n} p_{k}^{\frac{1}{1+m}}\right]^{1+m} \\
& +\frac{1}{m+1}\left\{\left(\begin{array}{c}
m+1 \\
2
\end{array}\right) E\left(G^{m-1}\right)-\left(\begin{array}{c}
m+1 \\
3
\end{array}\right) E\left(G^{m-2}\right)+\cdots+(-1)^{m+1}\right\}
\end{aligned}
$$

provided the guessing strategy satisfies:

$$
p_{k+1}^{\frac{1}{1+m}} \leq \frac{1}{k}\left(p_{1}^{\frac{1}{1+m}}+\cdots+p_{k}^{\frac{1}{1+m}}\right), k=1, \ldots, n-1 .
$$

In 1997, Dragomir and Boztaş [17] obtained for any guessing sequence:

$$
\begin{aligned}
& \left|E(G)-\frac{n+1}{2}\right| \leq \frac{(n-1)(n+1)}{6} \max _{1 \leq i<j \leq n}\left|p_{i}-p_{j}\right|, \\
& \left|E(G)-\frac{n+1}{2}\right| \leq \sqrt{\frac{(n-1)(n+1)\left(n\|p\|_{2}^{2}-1\right)}{12}},
\end{aligned}
$$

where $\|p\|_{2}^{2}=\sum_{i=1}^{n} p_{i}^{2}$ and

$$
\left|E(G)-\frac{n+1}{2}\right| \leq\left[\frac{n+1}{2}\right]\left(n-\left[\frac{n+1}{2}\right]\right) \max _{1 \leq k \leq n}\left|p_{k}-\frac{1}{n}\right|,
$$

where $[x]$ is the integer part of $x$.

For other results on $E\left(G^{p}\right), p>0$ see also [18]. We mention only, by making use of Grüss inequality, one has for $p, q>0$ that

$$
\left|E\left(G^{p+q}\right)-E\left(G^{p}\right) E\left(G^{q}\right)\right| \leq \frac{1}{4}\left(n^{q}-1\right)\left(n^{p}-1\right) .
$$

The above result may be complemented in the following way (see for example [11]).

Theorem 3. With the above assumptions, we have the inequality

$$
\begin{aligned}
& \left|E\left(G^{p+q}\right)-\frac{1+n^{q}}{2} E\left(G^{p}\right)-\frac{1+n^{p}}{2} E\left(G^{q}\right)+\frac{1+n^{q}}{2} \cdot \frac{1+n^{p}}{2}\right| \\
\leq & \frac{1}{4}\left(n^{q}-1\right)\left(n^{p}-1\right) .
\end{aligned}
$$

for any $p, q>0$.

Applications for different particular instances of $p, q>0$ may be provided, but we omit the details.

The following result also holds [9]. 
Theorem 4. Assume $S_{n}(p), p>0$ denotes the sum of $p$-power of the first $n$ natural numbers, that is

$$
S_{n}(p):=\sum_{k=1}^{n} k^{p}
$$

If

and

$$
p_{i} \leq(\geq) \frac{1}{n} \text { for } i \leq\left\lfloor\frac{S_{n}(p)}{n}\right\rfloor^{1 / p}
$$

$$
p_{i} \geq(\leq) \frac{1}{n} \text { for } i \geq\left\lfloor\frac{S_{n}(p)}{n}\right\rfloor^{1 / p}+1
$$

where $\lfloor x\rfloor$ denotes the integer part of $x$, then we have the inequality

$$
E\left(G^{p}\right) \geq(\leq) \frac{1}{n} S_{n}(p) .
$$

We are able now to state the first reasult for the momments of guessing mapping that may be obtained by the use of the inequality (2.2).

Theorem 5. If the probability distribution $\left(p_{1}, \ldots, p_{n}\right)$ satisfies the assumption

$$
0<p_{m} \leq p_{i} \leq p_{M} \text { for any } i \in\{1, \ldots, n\},
$$

then one has the inequality

$$
\left|E\left(G^{p}\right)-\frac{1}{n} S_{n}(p)\right| \leq \frac{1}{4} \frac{\left(p_{M}-p_{m}\right)}{n^{p / 2+1}} \cdot \frac{n^{p}-1}{\sqrt{p_{m} p_{M}}} \cdot S_{n}(p) .
$$

In particular, for $p=1$, we have the inequality

$$
\left|E(G)-\frac{n+1}{2}\right| \leq \frac{1}{8} \frac{\left(p_{M}-p_{m}\right)}{\sqrt{n}} \cdot \frac{n^{2}-1}{\sqrt{p_{m} p_{M}}} .
$$

If one uses the other Grüss type inequality (2.10), then one may state the following result as well.

Theorem 6. If the probability distribution $\left(p_{1}, \ldots, p_{n}\right)$ satisfies the assumption (3.15), then one has the inequality

$$
\left|E\left(G^{p}\right)-\frac{1}{n} S_{n}(p)\right| \leq\left(\sqrt{p_{M}}-\sqrt{p_{m}}\right)\left(\sqrt{n^{p}}-1\right) \sqrt{S_{n}(p)} .
$$

In particular, for $p=1$, we have the inequality

$$
\left|E(G)-\frac{n+1}{2}\right| \leq\left(\sqrt{p_{M}}-\sqrt{p_{m}}\right)(\sqrt{n}-1) \sqrt{\frac{n(n+1)}{2}} .
$$




\section{References}

[1] Biernacki, M., Pidek, H. and Ryll-Nardzewski, C. (1950), Sur une inégalité entre des intégrales definies, Ann. Univ. Mariae Curie-Skolodowska, A4, 1-4.

[2] Andrica, D. and Badea, C. (1988), Grüss' inequality for positive linear functionals, Periodica Math. Hungarica, 19, 155-167.

[3] Dragomir, S. S. and Booth, G. L. (2000), On a Grüss-Lupaş type inequality and its application for the estimation of $p$-moments of guessing mappings, Math. Comm., 5, 117-126.

[4] Dragomir, S. S. (2002), Another Grüss type inequality for sequences of vectors in normed linear spaces and applications, J. Comp. Analysis $\&$ Appl., 4, 157-172.

[5] Dragomir, S. S. (2002), A Grüss type inequality for sequences of vectors in normed linear spaces, (Preprint) RGMIA Res. Rep. Coll., 5,

Article 9. (ONLINE: http://rgmia.vu.edu.au/v5n2.html)

[6] Dragomir, S. S. (2001), Integral Grüss inequality for mappings with values in Hilbert spaces and applications. J. Korean Math. Soc. 38, 1261-1273.

[7] Dragomir, S. S. (1999), A generalization of Grüss's inequality in inner product spaces and applications. J. Math. Anal. Appl. 237, 74-82.

[8] Cerone, P. and Dragomir, S. S. (2002), A refinement of Grüss' inequality and applications, RGMIA Res. Rep. Coll., 5(2002), Article 15.

[9] Cerone, P. and Dragomir, S. S. (2002), New inequalities for Čebyšev functional involving two n-tuples of real numbers and applications, (Preprint) RGMIA Res. Rep. Coll., 5, Article 4. (ONLINE: http://rgmia.vu.edu.au/v5n3.html)

[10] Dragomir, S. S. and Pečarić, J.(1989), Refinements of some inequalities for isotonic functionals, Anal. Num. Theor. Approx., 18, 61-65.

[11] Dragomir, S. S. (2002), A companion of the Grüss inequality and applications, RGMIA Res. Rep. Coll., 5, Supplement, Article 13.

[12] Fink, A. M. (1999), A treatise on Grüss' inequality. Analytic and geometric inequalities and applications, 93-113, Math. Appl., 478, Kluwer Acad. Publ., Dordrecht,

[13] Pečaric, J. (1980), On some inequalities analogous to Grüss inequality. Mat. Vesnik 4(17)(32), 197-202.

[14] Massey, J. L. (1994), Guessing and entropy, Proc. 1994 IEEE Int. Symp. on Inf. Th., (Trondheim, Norway, 1994), p. 204.

[15] Arikan, E. (1996), An inequality on guessing and its application to sequential decoding, IEEE Tran. Inf. Th., 42, 99-105.

[16] Boztaş, S. (1997), Comments on "An Inequality of Guessing and Its Applications to Sequential Decoding", IEEE Tran. Inf. Th., 43, 2062-2063.

[17] Dragomir, S. S. and Boztas, S. (1997), Some estimates of the average number of guesses to determine a random variable, Proc. 1997 IEEE Int. Symp. on Inf. Th., (Ulm, Germany, 1997), p. 159.

[18] Dragomir, S. S. and Boztaş, S. (1998), Estimation of arithmetic means and their applications in guessing theory, Math. Comput. Modelling, 28(10) (1998), 31-43.

[19] Pólya, G. and Szegö, Z. (1925), Aufgaben und Lehrsatze aus der Analysis, Vol. 1, Berlin 1925, pp. 57 and 213-214.

[20] Shisha, O. and Mond, B. (1967), Bounds on differences of Means, Inequalities I, Academic Press. Inc., New York, London, 293-308. 
School of Computer Science and Mathematics, Victoria University of Technology, PO Box 14428, MCMC 8001, Victoria, Australia.

E-mail: sever@matilda.vu.edu.au

http://rgmia.vu.edu.au/SSDragomirWeb.html 